\title{
Critical Features in the Development of Exercise-based Interventions for People with Huntington's Disease
}

\author{
Lori Quinn, ${ }^{1}$ Anne Rosser ${ }^{2,3}$ and Monica Busse ${ }^{4}$
}

1. Senior Research Fellow, School of Healthcare Studies, Cardiff University, UK; 2. Professor of Clinical Neuroscience and Honorary
Consultant Neurologist, University Hospital of Wales, Cardiff, UK; 3. Co-director, Brain Repair Group, Cardiff University, UK; 4. Senior Lecturer, School of Healthcare Studies, Cardiff University, UK

\begin{abstract}
Exercise and physical therapies are increasingly being considered as a lifestyle intervention in people with Huntington's disease (HD). In addition to possible effects on motor function, there may be benefits in mood, behaviour and cognition from early in the disease. Certainly exercise may also infer general health benefits and there are recent suggestions that exercise may indeed potentiate disease modification. This review provides an overview of a strategic approach to the development and evaluation of complex exercise interventions in this neurodegenerative disease with a view to informing future clinical trials.
\end{abstract}

\section{Keywords}

Huntington's disease, physical activity, exercise, rehabilitation, physiotherapy

Disclosure: The authors have no conflicts of interest to declare.

Acknowledgements: The authors would like to acknowledge the National Institute for Social Care and Health Research, Wales (Community based Exercise Therapies in Huntington's' Disease (COMMET-HD), the Huntingtons Disease Association (Neil Glendinning Research Grant: Task Training in Huntingtons Disease [TRAIN-HD]) as well as the Paul Jefferies Waters Bequest Fund for research in Huntington's Disease (Move to Exercise) for providing funding support for the above mentioned studies. They would also like to acknowledge all the research participants who have so willingly engaged in this work. They further acknowledge Professor Helen Dawes and her colleagues at Oxford Brookes University, with whom they work closely.

Received: 26 March 2013 Accepted: 12 April 2013 Citation: European Neurological Review, 2013;8(1):10-3

Correspondence: Monica Busse, School of Healthcare Studies, Cardiff University, Ty Dewi Sant, Heath Park, Cardiff, CF14 4XN, UK. E: BusseME@cardiff.ac.uk

Huntington's disease (HD) is a genetic disease with autosomal dominant transmission and is unique amongst neurodegenerative diseases in that predictive testing is available before clinical symptoms are evident. ${ }^{1}$ As such, individuals with a family history of HD can choose to be tested and can establish if they will eventually get the disease. Some individuals choose not to know whether they carry the gene for HD, or are too young to be tested and are therefore considered at risk. For both the premanifest (those with a positive gene test but not showing symptoms) and at risk groups (those who have not taken the genetic test), there is the potential to develop interventions that can change the course or delay the onset of the disease. There is some evidence to suggest that lifestyle factors, such as activity level and education, may impact age of onset.2,3 Many factors can influence age of onset and extensive research is being conducted into identifying biomarkers. In people who are at risk or who are premanifest, the potential for lifestyle management and exercise as an intervention is intriguing.

Even before the onset of clinical symptoms, in the so-called prodromal phase, there is a slowly progressive loss of brain volume and degeneration of the basal ganglia. ${ }^{4}$ Despite relatively widespread degeneration, clinical symptoms may not be evident for up to 10 years from the start of these neural changes. At this stage and in early onset $\mathrm{HD}$, the development of compensatory networks may account for the lack of overt clinical signs, as new connections are developed to take over from damaged ones. These networks have the potential to be facilitated by experience and environment. Furthermore, it has been suggested that motor training may be one approach that can help to drive compensatory neural networks, ${ }^{5}$ thus compensating for the failing brain. In addition to possible effects on motor function, these therapies also have the potential to affect mood, behaviour and cognition from early in the disease. ${ }^{6,7}$

Over the past 6 years, the Cardiff Physiotherapy Group has taken a staged approach utilising the Medical Research Council Framework 8 , to develop exercise and physical interventions for people with HD. Our group has consulted with users, lay bodies and healthcare professionals to develop structured exercise interventions and identify barriers and facilitators to exercise in this population. ${ }^{9}$ We conducted the first randomised, controlled feasibility study of a defined, short-term, home-based exercise intervention in people with $\mathrm{HD}^{10}$ and have recently completed a mixed methodology, randomised feasibility study and process evaluation of a supported community-based gym exercise and walking programme.11 Additional controlled feasibility studies are currently underway or in set up, each with the aim of further advancing our understanding of different modes of exercise. Here, we describe our work to date and put forth suggestions for critical features of exercise studies based on our experiences in designing and delivering interventions in this population.

\section{Design and Delivery of Exercise-based Interventions in Huntington's Disease - Cardiff Experiences \\ Home-based Programmes}

Move to Exercise was a pilot study designed to evaluate a purposedeveloped home exercise DVD in people with HD. ${ }^{12}$ In this study, 25 
subjects with early to mid-stage Huntington's disease were randomly allocated to either an exercise intervention $(n=13)$ or a control group $(n=12)$. Subjects in the exercise intervention group were asked to perform exercises at home three times a week for 8 weeks using an exercise DVD. The control group received their usual care. Adherence in the intervention group was calculated from exercise diaries and measures of gait, balance, function, level of physical activity and quality of life were evaluated. Eleven participants from the intervention and 10 from the control group completed the study. Mean adherence was $29.4 \pm 1.8$ for the 32 prescribed sessions. There were no related adverse events. Analysis of covariance revealed differences between groups in gait speed, balance, function and level of physical activity, but not quality of life as measured by the SF-36. Effect sizes were large (>0.8) for the majority of the outcomes.

One important finding to emerge from the Move to Exercise study was that while the programme resulted in positive outcomes in the intervention group, a key factor to participant's successfully completing the exercise was caregiver support. ${ }^{10}$ Provision of appropriate support can be crucial in maintaining adherence and this seems to be particularly true for people with neurodegenerative diseases. ${ }^{13}$ In the Move to Exercise study, this support took many forms, including encouragement, support and occasionally physical assistance to perform the exercises. Further work is now required to understand in depth how to best support people with HD to exercise, given their range of cognitive and behavioural impairments.

Another important factor that could have affected the outcomes in Move to Exercise was whether the benefits seen could have been simply the result of social contact, or whether there was a specific training effect. A brief review of exercise studies conducted in people with Parkinson's disease (PD) and Alzheimer's disease (AD) has highlighted this issue. Of 69 studies in PD $(n=51)$ and dementia $(n=18)$, only 39 included a comparator arm. Main controls were physical $(n=32)$ and social contact $(n=7)$. The potential beneficial effect of social contact cannot be overlooked in exercise or physiotherapy interventions in people with neurodegenerative disease, particularly when the outcomes are related to quality of life or cognition. While it is important to first establish proof of principle, it is crucial for more studies to include comparator arms to properly tease out any intervention effects.

One potential issue with a DVD-based exercise programme, such as Move to Exercise, is that it is not individualised or context specific to the person's home environment (an aspect known to be required to ensure consistent learning and carry-over of new motor skill). ${ }^{14}$ It may be that some people with HD (particularly in the mid-stages) would benefit more from intensive, one-to-one therapy, where adherence and safety can be assured and in addition individual progression specific to a person's particular clinical and social needs can be accommodated.

In order to address these issues, we designed a home-based, 1:1 intervention [TRAIN-HD; ISRCTN 94284668]. This ongoing study aims to examine the feasibility and potential benefit of a home-based, task-specific physiotherapy led exercise programme on mobility, physical functioning, health-related quality of life and individualised goals determined in collaboration with the client and therapist. The programme was designed to address some of the common areas of difficulties seen in individuals with HD, namely walking, sit to stand and upright balance control.
Delivering interventions in a person's home has two important benefits. First, it is likely to improve recruitment and adherence to the programme by eliminating the need for participants to travel to a centre for service delivery. Second, physiotherapy provided in the home setting is by nature context-specific and is likely to be more relevant and meaningful to the participant to promote more effective outcomes with improved goal setting. ${ }^{15,16}$ Although such an approach requires increased travel time for the therapist and possibly increased isolation for both the therapists and people with $H D$, providing therapy at home puts fewer burdens on the patient and their family. ${ }^{17}$

The benefits of a programme based on task-specific practice as a means to improve functional abilities and task performance have been documented in individuals with neurological conditions such as stroke and PD. ${ }^{18,19}$ Task-specific practice involves practice of a task such as walking, rising from a chair, or reaching - using repetitions, alterations of the environment and modification of the conditions of the task as a means of progressing task difficulty. ${ }^{20}$ In addition to functional improvements, task-specific practice has been associated with neuroplastic changes within the cortical and sub-cortical areas of the brain, 21,22 indicating learning has occurred.

\section{Gym-based Programmes}

While home-based programmes have many possible benefits, including eliminating need for participant travel, convenience for participants and caregivers, and flexibility as to when the exercise is performed, such programmes also have inherent limitations. The range of equipment available in the home is limited, and thus participants may lose interest in a programme more readily. Furthermore, it is difficult to confirm fidelity of intervention if it is not directly supervised and monitored with appropriate equipment. Finally, exercising in an environment outside the home, such as a gym-based setting, may be more appealing for some participants, secondary to the natural social interaction that happens.

In order to determine if a gym-based programme was indeed feasible for people with $\mathrm{HD}$, we designed a mixed-mode exercise programme for people with early-mid stage HD.11 This multi-centre controlled study (ISRCTN 59910670) evaluated a 12-week exercise programme that included a 1x/week supervised gym session of stationary cycling and resistance exercises and 2x/week independent home-based walking programme. Thirty-one participants (16 male) [mean (SD) age 50.4 (11.4) years] with mid stage HD were randomly allocated to intervention $(n=16)$ or control (usual care) $(n=15)$. Acceptability was determined from subjective reports of tolerability and physiological measures recorded during the gym sessions. Assessment of benefit (blinded) included measures of physical abilities, disease severity and quality of life (SF-36). The retention rate was $81 \%$ (nine of the 11 individuals who started the intervention completed it) and seven out of those nine attended more than $75 \%$ (9/12) of the gym sessions. There were no related adverse events and the intervention was well tolerated by most participants. The confidence interval for the effect observed for the mental component summary (MCS) score of the SF-36 between groups ([ $n=9$; intervention, $n=13$; control; effect estimate: $7,95 \% \mathrm{Cl}[0.4,13.7]$ ) suggests clinical benefit of the intervention. Moderate effect sizes for cognitive outcomes and measures of walking were also observed.

Information obtained from process interviews conducted as part of this study did indeed suggest that some of the participants were very happy 
to go to the gyms and enjoyed the social interaction the gym setting provided. ${ }^{23}$ However, some participants found it to be a threatening environment and had concerns about how they were perceived in public. In addition, significant numbers of individuals approached for this study during recruitment chose not to enrol when they were told the exercise was taking place in a gym. We therefore propose that in order to develop a successful exercise intervention that is acceptable to a wide number of people with $\mathrm{HD}$, it is important to include personal preferences in terms of exercise environment to facilitate participant uptake and adherence.

To this end, our group has now developed a protocol that is designed to test the feasibility and potential benefit of an aerobic exercise programme in people with very early stage HD. Similar work has been conducted in patients with mild cognitive impairment ${ }^{24}$ with promising results. Our proposed study (EXeRT) will incorporate the personal preferences of the participant in terms of location and mode of exercise, and individualised goal setting will be central to the programme. This trial has two arms: a) 12-week exercise training with aerobic and anaerobic training components (intervention group), and b) a control group asked to continue as normal.

The intervention group will complete a 45-minute structured exercise intervention three times per week and the intervention will take place either in a community-based gym, or in cases where access to a gym is restricted or is not the personal preference of the participant, the intervention will take place in the participant's home with exercise equipment provided. Regardless of whether exercise is undertaken in a gym or at the home, the session will follow a set programme that will include a 10-minute warm up and stretches, followed by up to 30 minutes of exercise (treadmill, elliptical trainer or stationary bike) within an aerobic zone. Considering the suggestion that people with HD may have a lower anaerobic threshold than healthy individuals, ${ }^{25}$ and furthermore the sedentary nature of people with $\mathrm{HD}$, we will initially train individuals at the lower end of the $75-85 \%$ of heart rate (HR) reserve range (peak $\mathrm{HR}$ minus resting $\mathrm{HR}$, where peak $\mathrm{HR}$ is determined by the incremental bike test). Rating of perceived exertion (RPE, CR10 scale) and HR will be monitored.

This study will incorporate increased exposure in accordance with frequency and intensity exercise prescription protocols, which extends upon our previous work in COMMET-HD. ${ }^{11}$ Our proposed intervention will be more frequent than previous interventions and will be closely monitored by trained therapists to ensure a specific training effect from exercise.

\section{Critical Features of Exercise Study Designs - Lessons Learned}

While research in the area of exercise and physical activity in HD is in its infancy, pilot and feasibility data from our recent studies has already yielded important knowledge, which can be translated and applied to future research. Here we provide a summary of the key study design considerations for exercise and physical activity studies in people with HD.

\section{Adherence}

Documenting adherence and understanding barriers and facilitators to adherence to exercise programmes is essential to effective translation of clinical research. In our studies to date, adherence has been the primary measure; if individuals do not or will not adhere to a programme, its utility in the real world is limited. Accurate adherence data is best obtained by direct observation and one-to-one sessions, such as those provided in our studies to date (allow for use of session logs documented by research staff). For home programmes, exercise diaries are often used. Utility of exercise diaries do depend, however, on participant self-report. They must therefore be easy to use and should ideally be reviewed at least weekly with the research staff. Reasons for missed sessions should also be documented.

\section{Outcome Measures}

There have been considerable efforts over the past 10 years to determine the best outcome measures for use in clinical trials in the HD population. Clinical rating scales such as the Unified Huntington's Disease Rating Scale (UHDRS) are subject to variability, however, and may not be sufficiently sensitive to detect changes in response to an exercise intervention. While traditional measures of health and fitness have potential, it is very important to develop an understanding of exercise-related mechanistic effects. Our experiences suggest that measures of health and fitness are important for establishing intervention fidelity and are best used in supporting measures of exercise adherence. Establishing whether it is possible to delay the onset or progression of disease by preventing structural or cellular changes that are inherent in the development of symptoms is a crucial target. Studies such as TRACK-HD, ${ }^{4}$ which aim to identify sensitive and specific markers of disease progression and specific in-depth studies of the effect of exercise on brain function in HD are extremely relevant and important in the ongoing development and conduct of exercise trials in HD.

\section{Use of Mixed Methodology}

Exercise is a complex intervention where the activity itself and the local, social and environmental contexts interact to produce the outcome that is measured. ${ }^{8}$ Mixed methods designs that incorporate both quantitative and qualitative methodologies, for example process interviews of the participants, as well as the assessors and intervention therapists are therefore extremely important in promoting an in-depth understanding and appropriate interpretation of the findings. Practical considerations about the trial design, as well as acceptability of the assessment and intervention procedures, are further relevant to support design on Phase III trials.

\section{Goal Setting}

While HD is a degenerative, neurological condition, the importance of setting goals that result in positive changes in participation, lifestyle or daily living scales cannot be overstated. Use of tools such as Goal Attainment Scaling (GAS) ${ }^{27}$ can help patients to identify a specific focus and purpose of an exercise routine. We have successfully utilised the GAS in TRAIN-HD, as a means to develop and evaluate a collaborative training programme. While GAS should not be utilised alone as a primary outcome measure, it can be a sensitive measure of change in complex interventions such as TRAIN, where the focus of the intervention is functional and task-specific training..$^{28}$ As Turner-Stokes points out, GAS provides a person-centred perspective and can support interpretation of results from standardised outcome measures.

\section{Choice of Exercise}

Based on our extensive consultations with people with HD and our prior research in this area, we know that patients with HD need flexibility in terms of access to exercise facilities. For people with $\mathrm{HD}$, behavioural and cognitive impairments, such as apathy, and difficulty in planning, organising, sequencing and prioritising their daily activities, can 
significantly impact on their ability to initiate and adhere to an exercise programme. ${ }^{29,30}$ Consideration of these disease-specific factors, as well as understanding personal preferences and how they may change with the presence of a disease or disability, ${ }_{1}^{31}$ is necessary to successful implementation of any exercise-based programme. We therefore suggest that future studies incorporate a structured, mixed delivery approach that can be modified according to personal needs to facilitate people with HD to engage in exercise. Even though there will be some differences in intensities or specificity of training, the important factor is that an increase in overall physical activity is achieved.

\section{Structure and Support to Help People with Engagement}

Due to the nature of this disease and the complex interaction of both motor and behavioural symptoms in people with $\mathrm{HD}$, we believe that a high level of structure and support is crucial for the successful uptake of exercise and physical activity intervention. Furthermore, this structure is important to facilitate the potential for those receiving the intervention to develop the skills to participate independently in an exercise programme on completion of a particular study. Indeed the promotion of self-efficacy and self-determined lifestyle changes in relation to physical activity are essential in supporting sustained exercise habits. ${ }^{32}$

\section{Optimal Dose Response}

To date, there is insufficient research available in the field to support suggestions of what the appropriate exercise dosemaybe.Our recentstudy inpeoplewithearlytomid-stagepeopleattending1x/weekgymsessionand 2x/week at home walking programme showed potential for benefit in cognition and walking endurance, with moderate effect sizes identified. It is therefore likely that a higher dose, or intensity, is required to effectuate change. This recommendation needs to be considered with caution, particularly as fatigue may be an issue for some people with HD. Qualitative reports obtained during our studies highlighted that some people with HD did report fatigue following the exercise intervention; these individuals required some individual modification of their programme to help them to manage their fatigue. Fatigue has not, as yet, been considered a primary symptom of HD and we argue that it is important to know how prevalent a feature fatigue in HD may be. The underlying aetiology of fatigue and whether it is secondary to excessive movements, or part of the HD phenotype as is accepted in multiple sclerosis, ${ }^{33}$ has yet to be determined. To this end, in future work we plan to measure fatigue specifically, both as an outcome measure and as a potential adverse effect of the exercise intervention.

\section{Forward-thinking - The Future of Physical Therapies in Huntington's Disease}

It is clear that the changing clinical needs of the person with HD require stratified treatment approaches; the ultimate goal for our group is to provide a range of evidenced-based intervention strategies for people at varying stages of $\mathrm{HD}$, based on their specific clinical presentations. Here we present some of the lessons that we have learned along the way that we believe are critical to the design of future trials. We further recognise the importance of mechanistic evaluation in future exercise studies. Contemporary brain imaging techniques including structural and functional magnetic resonance imaging as well as diffusion tensor imaging will be important in future studies. ${ }^{34-37}$

Before Phase III trials are implemented, we believe that exercise interventions need to be appropriately described for implementation and replication. Furthermore, there should be a systematic published body of literature in support of the intervention which provides not only indications of acceptability but also of potential benefit and cost savings. Considering the potential that exercise has both in terms of general health benefit and in disease modification, these interventions can clearly provide important quality of life benefits to people with this devastating disease, as well as a platform for future clinical trials, where exercise and physical activity are complementary to pharmacological and surgical interventions with the ultimate aim of disease modification.
Tibben A, Predictive testing for Huntington's disease, Brain Res Bull, 2007:165-71.

Trembath MK, Horton ZA, Tippett L, et al., A retrospective study of the impact of lifestyle on age at onset of Huntington disease, MOV Disord, 2010;25(10):1441-50.

Lopez-Sendon JL, Royuela A, Trigo P, et al., What is the impact of education on Huntington's disease?, Mov Disord, 2011;26(8):1489-95

4. Tabrizi SI, Scahill RI, Durr A, et al., Biological and clinical changes in premanifest and early stage Huntington's disease in the TRACK-HD study: the 12-month longitudinal analysis, Lancet Neurol, 2011:31-42.

Neurol, 2011:31-42.
Cramer SC, Sur M, Dobkin BH, et al. Harnessing neuroplasticity for clinical applications, Brain, 2011:1591-609.

McDonnell MN, Smith AE, Mackintosh SF, Aerobic exercise to improve cognitive function in adults with neurological disorders: systematic review, Arch Phys Med Rehabil, 2011;1044-52.

7. Garber CE, Blissmer B, Deschenes MR, et al., American College of Sports Medicine position stand. Quantity and quality of exercise for developing and maintaining cardiorespiratory, musculoskeletal, and neuromotor fitness in apparently healthy adults: guidance for prescribing exercise, Med Sci Sports Exerc, 2011:1334-59.

8. Craig P, Dieppe P, Macintyre S, et al., Developing and evaluating complex interventions: the new Medical Research Council guidance, BMJ, 2008;337:a1655.

9. Quinn L, Busse M, Khali H, et al., Client and therapist views on exercise programmes for early-mid stage Parkinson's disease exercise programmes for early-mid stage Parkinson's disease Khalil K, Quinn L, van Deursen R, et al., The adherence to the use of a home based exercise DVD in people with Huntington's disease: participants' perspectives, Physical therapy, 2012;92(1):69-82.

11. Busse ME QL, DeBono K, et al., A Randomized Feasibility Study of a 12-week Community-based Exercise Program in people with Huntington's Disease, I Neurol Physic Ther, in press.

Khali H, Quinn L, van Deursen R, et al., What effect does a structured home-based exercise programme have on people with Huntington's disease? A randomized, controlled pilot study,

\section{Clin Rehabil, 2013; Epub ahead of print.}

13. Winward C, Supporting community-based exercise in longterm neurological conditions: experience from the Long-term Individual Fitness Enablement (LFE) project, Clin Rehabil, 2011:579-87.

14. Hubbard IJ, Parsons MW, Neilson C, Carey LM, Task-specific training: evidence for and translation to clinical practice, Occup Ther Int, 2009:16(3-4):175-89.

15. Holmqvist $\mathrm{L}$, von Koch $\mathrm{L}$, Envionmental factors in stroke rehabilitation - being in hospital itself demotivates patients, $B r$ Med J, 2001;322(7301):1501-2.

16. Holmqvist $L$, von Koch $L$, de Pedro-Cuesta J, Use of healthcare impact on family caregivers and patient satisfaction of rehabilitation at home after stroke in southwest Stockholm, rehabilitation at home after strok
Rehab Med, 2000;32(4):173-79.

17. Baskett J, Broad J, Reekie G, et al., Shared responsibility for ongoing rehabilitation: anew approach to home-based therapy after stroke, Clinical Rehabilitation, 1999;13(1):23-33.

18. Nieuwboer A, Kwakkel G, Rochester L, et al., Cueing training in th home improves gait-related mobility in Parkinson's disease: the RESCUE trial, I Neurol Neurosurg Psychiatry, 2007:134-40.

19. Widen Holmqvist L, von Koch L, Kostulas V , et al., A randomized controlled trial of rehabilitation at home after stroke in southwest Stockholm, Stroke, 1998;29(3):591-7.

20. Duff S, Quinn L, Functional movement development across the life span, Cech D, Martin S (eds), Motor learning and motor control, Philadelphia: WB Saunders, 2002.

21. Adkins DL, Boychuk J, Remple MS, Kleim JA, Motor training induces experience-specific patterns of plasticity across moto induces experience-specific patterns of plasticity act
cortex and spinal cord, I Appl Physiol, 2006:1776-82.

22. Page S, Szaflarski J, Eliassen J, et al., Cortical plasticity following motor skill learning during mental practice in stroke, Neurorehab Neural Repair, 2009;23(4):382-8.

23. Debono K, Quinn L, Wasley D, Experiences of people with Huntington's Disease participating in a 12 week exercise Disability and Rehabilitation (in review).

24. Baker LD, Frank LL, Foster-Schubert K, et al., Effects of aerobic exercise on mild cognitive impairment: a controlled trial, Arch Neurol, 2010:71-9.
25. Ciammola A, Sassone J, Sciacco M, et al., Low anaerobic threshold and increased skeletal muscle lactate production in subjects with Huntington's disease, Mov Disord, 2011;26(1):130-7. Ross CA, Tabrizi SJ, Huntington's disease: from molecular pathogenesis to clinical treatment, Lancet Neurol, 2011:83-98. Turner-Stokes L, Goal attainment scaling (GAS) in rehabilitation: a practical guide, Clin Rehabil, 2009:362-70.

28. Turner-Stokes $L$, Goal attainment scaling and its relationship with standardized outcome measures: a commentary, J Rehabil Med, 2011;43(1):70-2.

29. Kirkwood SC, Su JL, Conneally P, Foroud T, Progression of symptoms in the early and middle stages of Huntington disease, symptoms in the early and

30. Hamilton JM, Salmon DP, Corey-Bloom J, et al., Behavioural abnormalities contribute to functional decline in Huntington's disease, I Neurol Neurosurg Psychiatry, 2003;74(1):120-2. . Banks G, Bernhardt J, Churilov L, Cumming TB, Exercise preferences are different after stroke, Stroke Res Treat, 2012;2012:890946.

32. Teixeira PJ, Carraça EV, Markland D, et al., Exercise, physical activity, and self-determination theory: a systematic review, int $\lrcorner$ Behav Nutr Phys Act, 2012;9:78.

33. Berger JR, Pocoski J, Preblick R, Boklage S, Fatigue heralding multiple sclerosis, Mult Scler, 2013; Epub ahead of print Bohanna I, Georgiou-Karistianis N, Sritharan A, et al., Diffusion tensor imaging in Huntington's disease reveals distinct patterns of white matter degeneration associated with motor and cognitive deficits, Brain Imaging Behav 2011;5(3):171-80.

35. Bohanna I, Georgiou-Karistianis N, Egan GF, Connectivitybased segmentation of the striatum in Huntington's disease: vulnerability of motor pathways, Neurobiol Dis, 2011;42(3):475-81. Georgiou-Karistianis N, Scahill R, Tabrizi SJ, et al., Structural MRI in Huntington's disease and recommendations for its potential use in clinical trials, Neurosci Biobehav Rev, 2013;37(3):480-90. Gray MA, Egan GF, Ando A, et al., Prefrontal activity in Huntington's disease reflects cognitive and neuropsychiatric disturbances: the IMAGE-HD study, Exp Neurol, 2013;239:218-28. 